\title{
aula abierta
}

www.elsevier.es/aulaabierta

\section{Análisis de la producción científica en Brasil sobre dificultades de aprendizaje: una revisión bibliométrica}

\author{
Mirela de Oliveira Figueiredo a,", Sheila Maria Mazera ${ }^{\mathrm{a}}$, Maria Luisa Guillaumon Emmel ${ }^{\mathrm{a}}$ \\ y Estrella Fernández Albab
}

a Universidade Federal de São Carlos, Brasil

${ }^{\mathrm{b} U n i v e r s i d a d}$ de Oviedo, España

INFORMACIÓN DEL ARTÍCULO

Historia del artículo:

Recibido el 19 de abril de 2013

Aceptado el 4 de diciembre de 2013

\section{Palabras clave:}

Dificultad de aprendizaje

Trastorno del aprendizaje

Problemas de aprendizaje

Revisión bibliométrica

\section{RE S U M E N}

El objetivo de este estudio ha sido analizar la producción científica disponible sobre dificultades de aprendizaje (DA) en Brasil, e identificar las posibles deficiencias teóricas y metodológicas derivadas de la falta de una definición universal del problema, la posible confusión y la generalización de las DA en este país. Se realizó una revisión sistemática de los artículos nacionales publicados entre los años 2001 y 2011 en las bases de datos SciELO, Lilacs y BVS Adolec Brasil, en función del año de publicación, diseño metodológico, participantes y área temática o tópico. En un primer momento se obtuvieron 2187 resultados, aunque sólo 167 de los artículos diseminados entre las 2187 salidas fueron analizados de acuerdo con los criterios de inclusión. En 2009 se observó el mayor número de publicaciones, un total de 23. El diseño metodológico utilizado con mayor frecuencia fue el descriptivo de tipo correlacional (43 estudios). En 141 artículos, los participantes de la investigación eran alumnos y el área temática más representada estuvo relacionada con las habilidades de lectura y escritura (27 estudios). Este trabajo ha contribuido a delimitar la investigación realizada en torno a las DA, en Brasil, en relación con los criterios de interés mencionados.

(C) 2013 Instituto de Ciencias de la Educación de la Universidad de Oviedo. Publicado por Elsevier España, S.L. Todos los derechos reservados.

\section{Scientific production on learning disabilities in Brazil: A literature review}

\section{A B S T R A C T}

The aim of this study was to analyse the available scientific literature on learning disabilities (LD) in Brazil, and to identify theoretical and methodological gaps arising from the lack of a universal definition of the problem that is due to a confusion and generalization of LD in this country. A systematic review was conducted using articles published nationally between 2001 and 2011 in the databases SciELO, Lilacs and VHL Adolec Brazil, according to the year of publication, study design, participants and subject area or topic. Initially, results were found in 2187, but only 167 articles were analysed in accordance with the inclusion criteria. The highest number of publications was seen in the year 2009, with a total of 23 . The methodology used most often was descriptive and correlational (43 studies). In 141 articles the survey participants were students, and the subject area represented was more related to reading and writing ( 27 studies). This work has contributed to presenting a national overview on the study of learning difficulties in relation to the interest criteria mentioned.

(C) 2013 Instituto de Ciencias de la Educación de la Universidad de Oviedo. Published by Elsevier España, S.L. All rights reserved.

\footnotetext{
*Autor para correspondencia: Mirela de Oliveira Figueiredo. Universidade Federal de São Carlos. Rua Boa Morte, 1246. 13480, Limeira (Brasil). Correo electrónico: mirelafigueiredo@hotmail.com
}

\section{Introducción}

En Brasil no hay en la actualidad una definición única sobre el concepto de dificultades de aprendizaje. Debido a la heterogeneidad de los síntomas, aún hay confusión y desconocimiento sobre el tema. Esto ha hecho que las dificultades de aprendizaje (DA), erróneamente, se vinculen únicamente al fracaso escolar, caracteriza- 
do por la deserción académica y la repetición de cursos (Moojen, 2012; Sisto, 2001).

En este estudio se consideran las DA según la definición promovida por el National Joint Committee on Learning Disabilities (1994, 2011) y por Correia (1991, 2004, 2007), que desde hace décadas han llevado a cabo investigaciones e intentado proponer una definición apropiada para los Estados Unidos y Portugal, respectivamente.

La definición de dificultades de aprendizaje pasó a ser utilizada internacionalmente en el inicio de la década de 1960. Esto ocurrió dada la necesidad de comprender por qué un grupo de alumnos, con capacidad intelectual en torno a la media o por encima de ésta (cociente intelectual $[\mathrm{CI}]$ medio 100 ) presentaba fracaso escolar, especialmente ante tareas de lectura, escritura y/o cálculo.

Según el Manual Diagnóstico y Estadístico de Trastornos Mentales (DSM-IV-TR) (American Psychiatric Association, 2002), las DA se incluyen en el apartado de trastornos del aprendizaje y se clasifican como sigue: trastorno de la lectura (F81.0), trastorno en matemáticas (F81.2), trastorno de la expresión escrita (F81.8) y trastorno del aprendizaje no especificado (F81.9). En el DSM-IV-TR se utiliza el término trastornos del aprendizaje y se establece que éstos pueden diagnosticarse cuando los resultados de un alumno en diferentes pruebas estandarizadas e individualmente aplicadas (de lectura, matemáticas o expresión escrita) están sustancialmente por debajo de lo esperable para su edad, nivel de escolarización o capacidad intelectual.

De acuerdo con la literatura científica, los alumnos con DA presentan trastornos que interfieren en la recepción, integración, retención o expresión de la información, lo que implica problemas de memoria, perceptivos, motores, de lenguaje, de pensamiento y/o metacognitivos que generan una limitación o impedimento para el aprendizaje de la lectura, escritura, cálculo y aptitudes sociales (Correia, 1991, 2007; National Joint Committee on Learning Disabilities, 1994). Las causas de estas dificultades deben estar relacionadas con la persona que aprende y el ambiente físico y/o social de la escuela y no producirse como resultado de situaciones de privación sensorial, deficiencia intelectual y/o física. Capellini et al. (2007) manifiestan que las DA acarrean bajo rendimiento, retraso en el aprendizaje y que, por tanto, pueden promover entre los alumnos el abandono escolar.

Correia (2004) apunta a la existencia de seis categorías de DA ya identificadas: auditivo-lingüística, viso-espacial, motora, organizacional, académica y socioemocional. Sin embargo, aun con la existencia de definiciones e indicadores para la detección de las DA, el alumno con dificultades pasa constantemente por interpretaciones que tienden a simplificar la gravedad de sus déficits, siendo éstos relacionados con condicionantes socioeconómicos, familiares y/o culturales, lo que genera en ocasiones, y en un sentido amplio, la elaboración de un cuadro clínico acerca de sus necesidades, erróneo. En otras ocasiones, el alumno puede ser confundido con un estudiante con problemas para aprender derivados de una capacidad intelectual inferior a la media (Correia, 2004). De hecho, en Brasil, el número de alumnos que no consiguen superar con éxito los cursos académicos ha aumentado, siendo estos estudiantes diagnosticados, en muchas ocasiones, como alumnos con discapacidad intelectual o con problemas de comportamiento (Bandeira, Silva, Magalhães, Pereira y Del Pettre, 2006; Marturano y Loureiro, 2003; Moojen, 2012).

La actual Política Nacional de Educación Especial considera que el alumno con necesidades educativas especiales (NEE) es aquel con una discapacidad intelectual, física o sensorial, un trastorno generalizado del desarrollo o una alta capacidad/sobredotación (Ministério da Educação, 2007). No obstante, las NEE son las necesidades de cualquier niño que presenta o está en riesgo de presentar dificultades en su proceso de escolarización. Así, el término alumnos con NEE abarca a todos aquellos estudiantes que se enfrentan a un posible fracaso escolar como consecuencia de diferentes razones que pueden estar impidiendo su progreso académico (Ferreira, 2006). Aun así, no hay una estimación concluyente, tanto en el censo del Ministerio de Educación y Cultura brasileño como en la literatura científica, sobre cuántos niños con DA están escolarizados en centros públicos de este país.

Además, en Brasil, los alumnos con DA aun siendo considerados como estudiantes con NEE (Hallahan y Kauffman, 2005) no tienen las mismas condiciones legales para permanecer escolarizados $\mathrm{y}$, por tanto, no cuentan con acceso a los recursos humanos y materiales adicionales que podrían estimular un aprendizaje eficaz; por ejemplo, aulas específicas o personal especializado de apoyo en las clases ordinarias.

Se entiende que el punto de partida de esta circunstancia puede situarse en la falta de una definición universal de las DA, que a su vez lleva vinculado el desconocimiento, confusión y generalización del problema y con ello un déficit en la estimación del número de alumnos con DA en Brasil. Por ello, se considera importante la profundización en el estudio de la investigación realizada en torno a las DA para lograr, en última instancia, que los estudiantes con trastornos y dificultades del aprendizaje tengan derecho a un acceso igualitario a una educación de calidad y al apoyo necesario. Es imprescindible, por tanto, la adopción de políticas eficaces para promover su éxito académico. Se considera que la formulación de políticas adecuadas requiere que los representantes del gobierno y los líderes educativos comprendan la naturaleza de las DA y las necesidades de estos estudiantes utilizando, por ejemplo, los datos de la investigación empírica en el área de base. Así pues, el objetivo de este trabajo ha sido realizar una revisión bibliométrica de los artículos publicados sobre las DA en Brasil, en la última década, para caracterizar la producción científica brasileña sobre el tópico y favorecer la identificación de posibles lagunas teóricas y metodológicas en el área de las DA que puedan estar en la base de la falta de consenso teórico sobre éstas y la estimación de la prevalencia de alumnos con estas dificultades en las aulas brasileñas.

\section{Método}

\section{Unidad de análisis}

En esta revisión solamente se han tenido en cuenta artículos científicos recogidos en diferentes bases de datos que acogen publicaciones relacionadas con las DA y realizadas en Brasil. No se han tenido en consideración otras publicaciones como, por ejemplo, libros o capítulos de libros y artículos que no estuvieran escritos en lengua portuguesa. En un primer momento, se extrajeron 2187 resultados posibles mediante las búsquedas realizadas en las bases de datos seleccionadas y con las palabras clave elegidas. Tras el proceso de eliminación de los artículos que no cumplían los criterios de inclusión, los estudios objeto de análisis para esta revisión se redujeron a un total de 446. Puesto que existen numerosos artículos que se repiten en más de una base de datos, incluso dentro de la misma base al introducir dos o más palabras clave diferentes, se identificaron los artículos repetidos considerándolos para el análisis una sola vez. Así, con la exclusión de artículos que no cumplían los criterios de inclusión y la eliminación de los artículos repetidos entre los resultados observados en un primer momento, al final se trabajó en este estudio con un total de 167 artículos, los cuales fueron analizados minuciosamente en cuanto al año de publicación, diseño metodológico, participantes, y objetivo de la investigación.

\section{Procedimiento}

Para buscar los resúmenes de artículos publicados, se seleccionaron las bases de datos SciELO (Scientific Electronic Library Online), Lilacs (Literatura Latinoamericana en Ciencias de la Salude) y BVS 
Adolec Brasil. Todas estas bases recogen publicaciones electrónicas y fueron elaboradas teniendo en cuenta las necesidades de comunicación científica entre los países en desarrollo, especialmente de América Latina y Caribe. La base de datos Lilacs publica específicamente investigaciones relacionadas con el ámbito de la salud, y BVS Adolec Brasil sobre el ámbito de la salud de adolescentes y jóvenes de Brasil. La búsqueda realizada en las mencionadas bases de datos se efectuó durante los meses de enero y febrero de 2013.

El uso de bases de datos con perfiles de publicación distintos se debe a la interacción que se produce entre el estudio de la salud y la educación cuando el objeto de investigación se centra en las causas de aparición de DA. En la actualidad, el concepto de salud implica, además de condicionantes médico-sanitarios, dimensiones sociales, culturales, espirituales y educativas relacionadas con los alumnos y con la comunidad en la que viven (Rangel, 2009). Para Bastos y Deslandes (2008), Silva y Dessen (2001) y Werner (1998), la consideración de los contextos ambientales y sociales es de capital importancia para la comprensión de las causas de las DA. Correia (2007) enfatiza la relevancia de las características del alumno que aprende, es decir, del estudio de los déficits derivados de las alteraciones en el funcionamiento cerebral y de la forma en la cual el cerebro procesa la información, o sea, de lo que se aprende (Carrera, 2009; Feldman, 2005). Otros autores priman los aspectos psíquicos como causa subyacente en las DA, es decir, subrayan la importancia de quién aprende. Por último, también se estudian las condiciones externas que se refieren a aspectos más sociales, es decir, cómo se aprende y al ambiente de aprendizaje dónde se aprende. En este sentido, para poder analizar la investigación publicada en torno a las DA se consideró necesario analizar bases de datos que abarcaran todas la dimensiones de interés citadas.

Las palabras clave utilizadas fueron: dificultad de aprendizaje [ $d i$ ficuldade de aprendizagem/aprendizado], dificultades de aprendizaje [dificuldades de aprendizagem], trastornos del aprendizaje [transtorno de aprendizagem] y problemas de aprendizaje [distúrbio de aprendizagem]. Se escogieron estos descriptores ya que son los términos adoptados por la comunidad científica en el ámbito de la investigación de las dificultades del aprendizaje.

Los criterios de inclusión y exclusión de las publicaciones adoptados fueron los siguientes: a) artículos publicados en revistas nacionales, excluyéndose otras publicaciones como libros, comunicaciones en congresos, trabajos de investigación y tesis, reseñas o relatos; b) estudio de DA en alumnos de enseñanza básica o media sólo con esta problemática, excluyendo estudiantes de otros niveles y condiciones (universitarios, trabajadores) y estudiantes con DA como consecuencia de alguna discapacidad o deficiencia (intelectual, orgánica y/o física); $c$ ) artículos escritos en portugués; $d$ ) los artículos que se encontraban en más de una base de datos sólo fueron tenidos en cuenta una vez, y e) artículos publicados en la última década (entre los años 2001-2011).

Para registrar los datos se utilizó un protocolo de registro que hizo posible organizar la información de cada artículo localizado a partir de los descriptores empleados. En este protocolo se registraron los siguientes puntos: tipo de diseño metodológico del estudio, objetivo del estudio, descripción de los participantes, año de publicación y bases de datos de referencia.

Para definir el diseño metodológico se utilizaron los parámetros de Goodwin (1995) y Thomas y Nelson (1996). En líneas generales, estos autores contemplan la existencia de diseños analíticos, descriptivos, experimentales, cuasi-experimentales y cualitativos. Las investigaciones con diseño analítico implican el estudio y evaluación de la información disponible en un intento de explicar el contexto de un fenómeno o tópico, siendo categorizadas en históricas, filosóficas, de revisión o metaanálisis. Los estudios descriptivos se caracterizan por identificar el estado de la investigación, recoger opiniones o proyecciones futuras con respecto a un tópico, y se dividen en normativos, correlacionales, estudios de caso, longitudinales o trans- versales. La investigación experimental lleva tras de sí un método de investigación que implica la manipulación de tratamientos en un intento de establecer relaciones causa-efecto en las variables investigadas, mediante el control cuidadoso y completo del diseño. En la investigación cuasi-experimental, el investigador intenta preparar un diseño que contemple una aproximación al mundo real, intentando controlar, de la mejor forma posible, algunos condicionantes que afecten a la validez interna del estudio. Por último, las investigaciones cualitativas implican la observación intensiva y durante un largo período en un ambiente natural. El registro de los fenómenos observados en su ambiente es preciso y detallado, y la interpretación y análisis de los datos se realiza a través de descripciones y narrativas. Estas investigaciones pueden ser etnográficas, interpretativas, de investigación-acción, fenomenológicas.

Con respecto a los participantes de las publicaciones, se consideraron las categorías: estudios realizados con alumnado (con edades que variaban de los 5 a los 14 años), estudios sobre profesorado, estudios sobre las familias, así como cualquiera de sus combinaciones.

En cuanto al tópico central de los estudios se recogieron 15 categorías a partir de una lectura previa de los resúmenes de los artículos, identificando puntos de convergencia entre los tópicos encontrados: conciencia fonológica; desarrollo cognitivo-lingüístico; desarrollo psicomotor, sensorial o perceptivo; autoconcepto y autoeficacia; habilidades de lectura y escritura; problemas comportamentales y DA; representaciones sobre el alumno y su rendimiento; orígenes históricos de la medicalización en DA; procesamiento numérico, de cálculo y memoria operacional; procesamiento auditivo; perfil ortográfico; evaluación asistida; intervenciones terapéuticas/psicopedagógicas; aspectos neurobiológicos y causas de las DA, e instrumentos de evaluación (evaluación/fiabilidad).

\section{Análisis de datos}

Tras la selección de los artículos relevantes para este estudio y a través del análisis pormenorizado de los resúmenes, se organizó la información en diferentes categorías de acuerdo con el protocolo de registro. Muchos de los resúmenes de los artículos analizados no contenían toda la información necesaria para comprender el estudio, lo que dio lugar a errores de interpretación acerca de la metodología empleada, el tópico central del artículo o el tipo de participantes de la muestra. En algunos casos, por tanto, fue necesario revisar el artículo íntegramente para poder satisfacer las demandas del presente estudio. Dado el carácter exploratorio de éste, se procedió a analizar los datos a través de diferentes estadísticos descriptivos (frecuencias y porcentajes) utilizando el paquete estadístico SPSS.19.

\section{Resultados}

En relación con las bases de datos de referencia, el mayor número de artículos utilizados para la revisión, antes de descargar los trabajos repetidos, se obtuvieron de la base de datos Lilacs (321 artículos), seguida de la base de datos SciELO (102 artículos) y, por último, Adolec Brasil (23 artículos). Con respecto a las palabras clave que facilitaron la búsqueda de artículos, cabe reflejar que la palabra clave dificultades del aprendizaje (145 artículos) fue la que facilitó la recuperación de un mayor número de estudios, seguida de trastornos del aprendizaje (110 artículos) y dificultad del aprendizaje (96 artículos). Las palabras clave con menor impacto en la búsqueda fueron dificultad en el aprendizaje (55 artículos) y problemas de aprendizaje (41 artículos).

En cuanto al año de publicación de los artículos, se observa un crecimiento de las publicaciones sobre DA, siempre de acuerdo con los criterios de inclusión y exclusión del presente estudio, a lo largo de los años. Así pues, en los 5 años de inicio de la década revisada 
(2001-2005) se publicaron el 29\% de los 167 artículos analizados en el presente trabajo. Durante los 3 años siguientes (2006-2008), el $31 \%$ del total de artículos, porcentaje que aumenta (40\%) cuando el análisis se realiza en relación con los tres últimos años de la década de referencia. El mayor número de publicaciones se concentra entre los años 2009 y 2011 (fig. 1).

En cuanto al tipo de diseño, se observa que el diseño metodológico adoptado con mayor frecuencia fue el descriptivo, utilizado en el $52 \%$ de las 167 publicaciones totales. Del total de publicaciones con un diseño descriptivo, aproximadamente el $50 \%$ de éstas presentaba un diseño descriptivo de tipo correlacional, es decir, buscaban explorar relaciones entre las variables objeto de estudio. Un 15\% utilizaba un diseño descriptivo de tipo normativo, intentando esclarecer las diferencias entre edades y sexo en relación con las habilidades, rendimiento o aptitudes de los alumnos. Otro 15\% utilizaba un diseño descriptivo de tipo analítico, con la intención de recoger información sobre la práctica u opiniones actuales en relación con una población específica. Un $14 \%$ mostraba un diseño descriptivo de tipo transversal, cuyo objetivo se centraba en analizar los cambios de comportamiento visibles a través de los años, con muestras de niños de diferentes edades, para proporcionar información sobre los efectos de maduración. Se contó con un número muy limitado de estudios de caso ( $5 \%$ del total), y sólo con un estudio de tipo longitudinal (1\%) que pretendía analizar los cambios de comportamiento visibles en un mismo grupo de niños a través de los años y por un período determinado (fig. 2).

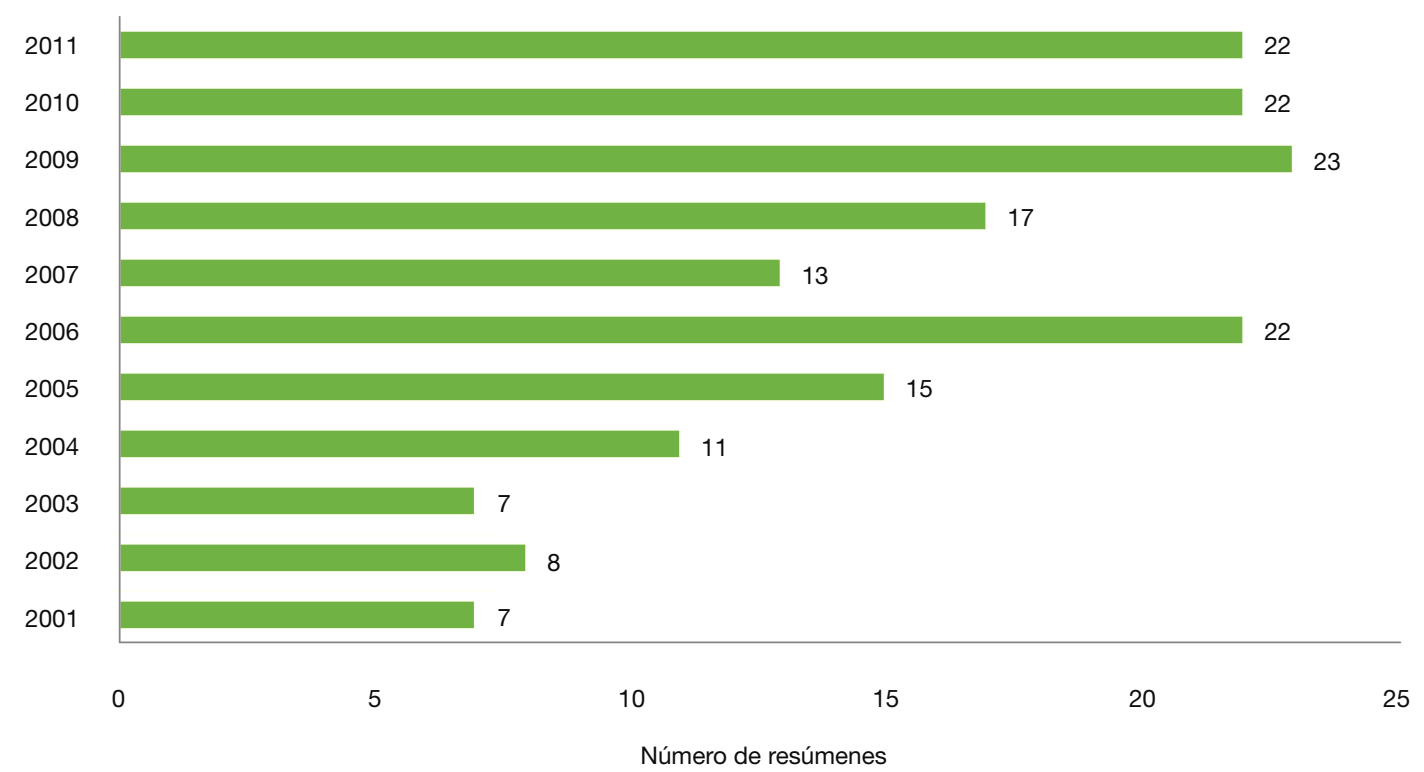

Figura 1. Distribución de los artículos seleccionados para el análisis en función del año de publicación.

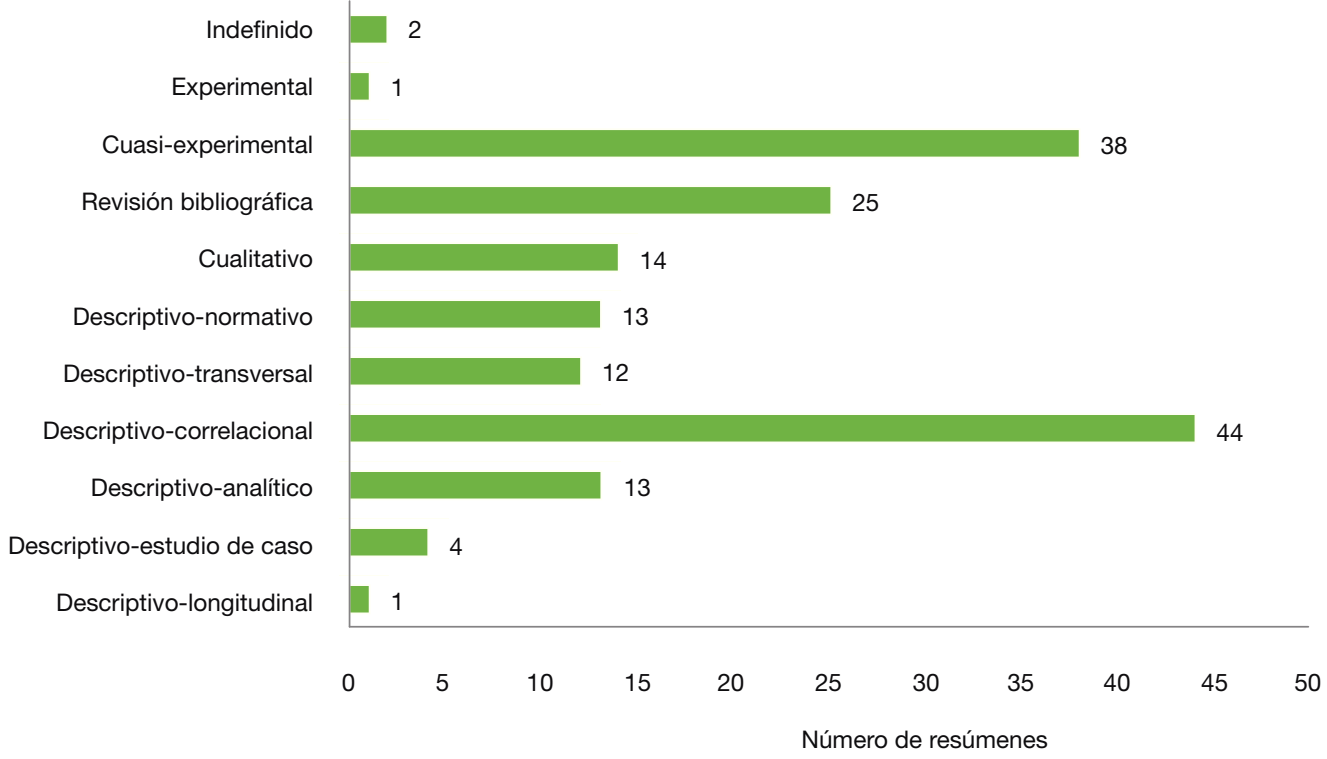

Figura 2. Distribución de los artículos seleccionados para el análisis en función del tipo de diseño metodológico. 
En segundo lugar, tras los diseños descriptivos, el diseño utilizado en mayor medida fue el diseño cuasi-experimental (23\% de los 167 artículos). En estas investigaciones se realizaron diferentes intervenciones con el fin de establecer la relación causa-efecto en las variables estudiadas, buscando controlar los factores que afectan a la validez interna y hacer que el entorno de la investigación se parezca, lo más cercano posible, al mundo real. El 15\% del total de los estudios analizados eran revisiones bibliográficas. Este tipo de investigación intenta analizar, críticamente, la producción reciente de un área en particular, incluyendo el análisis, evaluación e integración de la literatura publicada. El 8\% de las publicaciones presentaban un diseño cualitativo. Este tipo de estudios se caracterizan por el uso de la observación, intensiva y durante largos períodos, de un suceso en su ambiente natural con el fin de encontrar significados inherentes a determinadas creencias, actitudes, hábitos. Por último, sólo se encontró un estudio con un diseño de tipo experimental. Este tipo de diseño consiste en la manipulación de algún tratamiento para poder establecer relaciones causa-efecto en las variables investigadas, contando con la manipulación de la variable independiente para analizar su efecto sobre las variables dependientes (fig. 2).

En cuanto a los participantes, la mayoría de los artículos recuperados centran su atención en el alumno. En total, el 84\% de los artículos utilizaron muestras de alumnos de entre 5 y 14 años (fig. 3).

Por último, los tópicos de investigación preferentes recogidos en los estudios analizados se muestran en la figura 4, donde se ofrece también una visión acerca de los subtemas preferentes para los in-

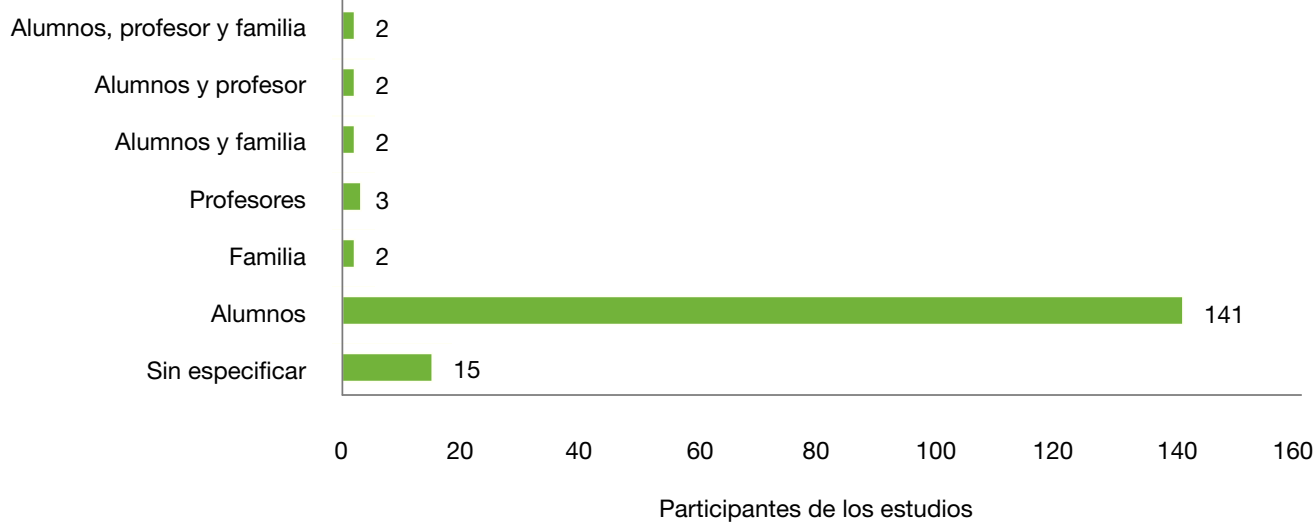

Figura 3. Tipo y número de participantes de los artículos seleccionados para la revisión.

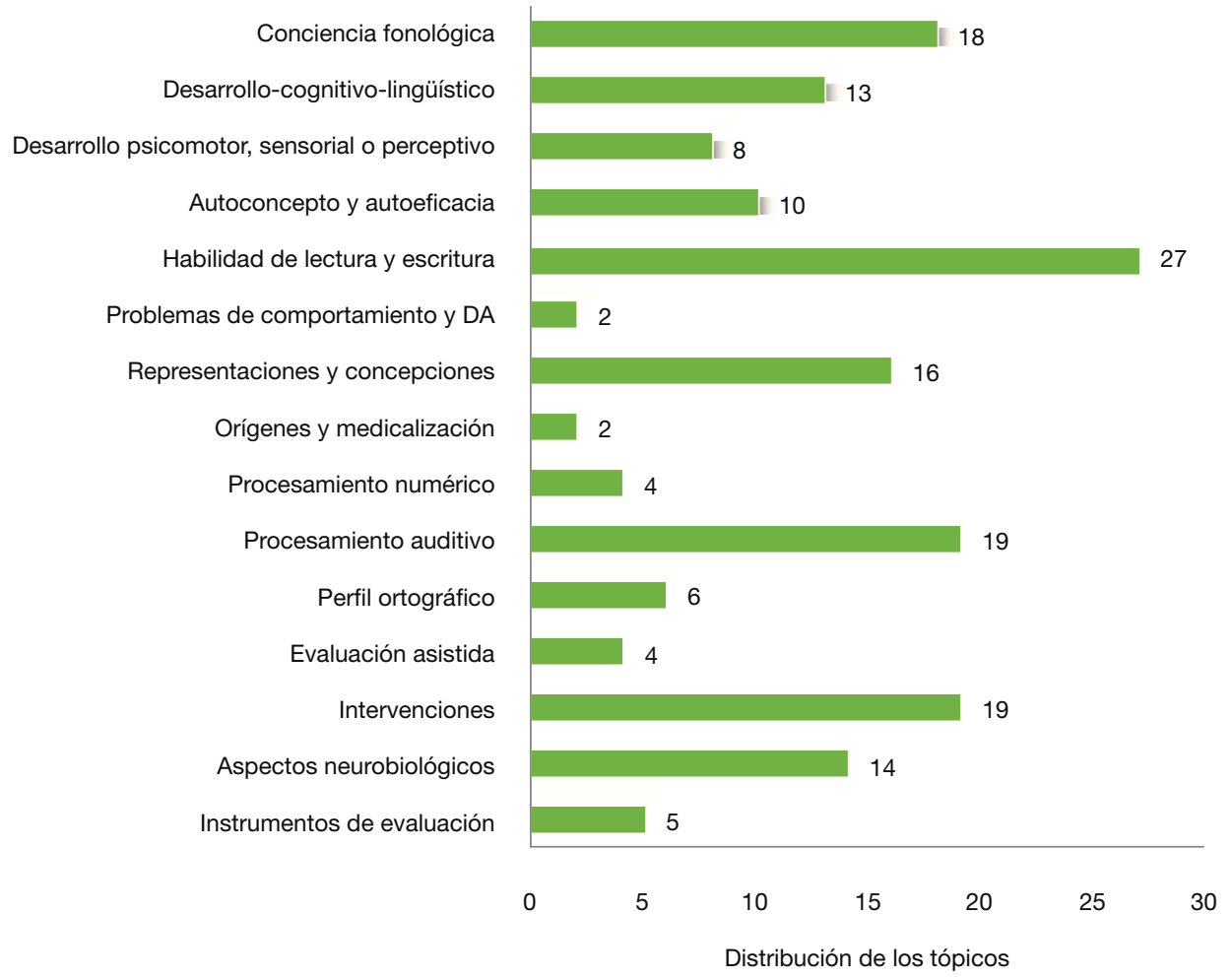

Figura 4. Distribución de los estudios según el tópico de interés de los mismos. 
vestigadores en el ámbito de las DA. De entre las investigaciones recuperadas, el tópico habilidades de lectura y escritura fue el más estudiado (16\% de las 167 publicaciones totales). Le seguían los tópicos procesamiento auditivo (considerando dentro de este grupo los artículos que centraron su estudio en la descripción o intervención sobre el procesamiento auditivo y relacionándolo con el rendimiento en la lectura y escritura), intervenciones (teniendo en cuenta cualquier tipo de intervención en DA y/o aspectos relacionados como creatividad, enfoques o habilidades sociales) y conciencia fonológica (estudio de su relación con el desempeño en lectura y escritura), todos ellos representados en porcentajes similares (aproximadamente el 11\%). Por otro lado, cabe destacar que, además de los mencionados, el $8 \%$ de los artículos centraron su estudio en el desarrollo cognitivo y lingüístico, o sea, en la asociación de componentes del lenguaje oral con el proceso cognitivo del aprendizaje de la lectura y escritura, y otro $4 \%$ en el perfil ortográfico, es decir, se interesaban por el estudio del proceso de adquisición ortográfica en relación con la lectura y la escritura. Así pues, 83 artículos (de 167) se centraron en el estudio de los requisitos necesarios para aprender a leer y escribir. Por el contrario, sólo se encontraron cuatro investigaciones centradas en el estudio del procesamiento numérico, de cálculo y memoria operacional. El 5\% de los estudios se centraron en los procesos de evaluación, analizando la validez y fiabilidad de diferentes instrumentos de evaluación y profundizando en el estudio de la evaluación asistida, que representa una modalidad de evaluación diferente y complementaria a la evaluación tradicional de habilidades cognitivas y lingüísticas. En este tipo de evaluación, el evaluador ayuda al alumno durante el proceso de resolución de los ítems, tras una fase inicial sin ayuda, y es común en aquellas investigaciones donde el propio sujeto actúa como control de su propia evaluación, es decir, se evalúa inicialmente al sujeto, se evalúa con ayuda y, posteriormente, se vuelve a evaluar sin ayuda. En cuanto a la etiología de las DA, el 10\% de los trabajos se centraron en los aspectos neurobiológicos y causas de DA (epidemiología, etiología, descripción del cuadro clínico, comorbilidad, diagnóstico y factores de riesgo relacionados con las DA) y en los orígenes y medicalización en DA (analizando las disfunciones neurológicas como generadoras de las DA). El resto de publicaciones se centraron en el estudio de los problemas de comportamiento y DA, el autoconcepto y autoeficacia (estudio de la relación existente entre las creencias que el alumno posee sobre sí mismo y cómo pueden afectar a su proceso de aprendizaje), el desarrollo psicomotor, sensorial y perceptivo (análisis del desarrollo de las funciones relacionadas con la coordinación motora fina o general, esquema corporal, organización espacio-temporal), y representaciones sobre el alumno y su rendimiento (estudio de las concepciones que padres y profesores tienen sobre el alumno y las expectativas relacionadas con las dificultades del aprendizaje).

\section{Discusión y conclusiones}

Actualmente, en Brasil, no parece existir un consenso en relación con la definición e intervención sobre las DA. Por tanto, este trabajo ha centrado su interés en el estudio de la literatura científica nacional (Brasil) acerca de este tópico con el fin de ofrecer una visión general del mismo en el ámbito de la investigación que pueda ser transferible a todos los ámbitos de actuación, por ejemplo, intervención o políticas de educación.

Según Moojen (2012), los términos dificultad de aprendizaje, trastorno del aprendizaje y problemas de aprendizaje se han utilizado al azar a la hora de emitir diagnósticos, tanto en la literatura científica como en la práctica clínica o escolar. Para França (1996) el término dificultades de aprendizaje enfatiza los aspectos pedagógicos y socioculturales del problema, es decir, la importancia de variables intervinientes ajenas al propio alumno, pero relacionadas con su proceso de estudio. Sin embargo, trastornos del aprendizaje sitúa la problemática exclusivamente en el propio estudiante, sugiriendo, por ejemplo, la existencia de deterioro neurológico.

Considerando que el término utilizado en uno de los manuales diagnósticos más comúnmente utilizados, el DSM-IV-TR, es trastornos del aprendizaje (American Psychiatric Association, 2002), los resultados obtenidos apuntan a una falta de estandarización en cuanto a la terminología adoptada. Ciertamente, unos términos se adoptan más que otros por cuestiones epistemológicas en cada área de investigación. Sin embargo, adoptar una definición y terminología consensuadas entre la comunidad en general y, en concreto, entre investigadores, políticos, médicos y educadores de un país, hace posible la "operacionalización" de acciones y decisiones que aseguren a los alumnos con DA una educación de calidad y con respuesta eficaz a sus necesidades reales.

Tras el análisis de los artículos seleccionados, en relación con la distribución de los estudios en función del diseño metodológico, parece que la tendencia es utilizar diseños descriptivos, tendencia que ya fue reflejada por Mastropieri et al. (2009) en una revisión precedente realizada sobre revistas de educación especial. No obstante, según los artículos analizados, parece observarse un aumento sustancial del uso de diseños cuasi-experimentales en la última década. Esto concuerda con el movimiento, surgido hace algunos años en el plano internacional, que defendía la necesidad de que las decisiones de política educacional y de las prácticas pedagógicas deberían basarse en indicadores producidos por investigaciones de corte experimental (Campos, 2009). No obstante, el número de estudios experimentales recogidos en este trabajo no siguen la línea de este movimiento. Mastropieri et al. (2009) apuntan a que el número tan reducido de publicaciones con este diseño puede justificarse por el grado de complejidad que supone su uso, como la exigencia de control de las variables o la aleatorización de los sujetos, algo muy poco común en contextos académicos donde se suele trabajar con grupos naturales ya formados. Por ello, es más factible el uso de investigaciones cuasi-experimentales. Sin embargo, aunque no se observa un aumento sustancial de investigaciones basadas en diseños experimentales, sí que se produjo una expansión del uso de las investigaciones cuasi-experimentales, que aportan cierto grado de robustez a la investigación y no requieren de largos períodos de observación y recogida de datos, aleatorización de los sujetos y considerando criterios de control de las variables más flexibles (Shadish, 2001). También se observa un número razonable de estudios de revisión. Según Cabral-Filho (2006), una de las causas de la publicación de un gran número de este tipo de trabajos podría estar relacionada con las investigaciones realizadas por los posgraduados, ya que ese tipo de estudios cubren a su vez las exigencias planteadas a los alumnos para superar sus cursos de posgrado, al menos en Brasil. Este hecho, según Cabral-Filho (2006), representa una política científica, saludable, que pude tener muchos beneficios para el país. Sin embargo, el amplio número de estudios de revisión también puede estar apuntando a la constante necesidad de los investigadores de fundamentar, teóricamente, el objetivo de sus estudios (Rother, 2007), siendo necesario para ello la existencia de revisiones que representen el conocimiento adquirido hasta el momento en relación con esos objetivos (Cook, Mulrow y Haynes, 1997; Higgins y Green, 2011). Por otro lado, se observa un reducido número de publicaciones cualitativas. Esto puede justificarse si se tiene en cuenta que el uso de este tipo de diseño es más reciente. Turato (2005) apunta a que los trabajos con este tipo de diseño, en épocas anteriores, no eran considerados científicos, por lo que generalmente eran rechazados en el proceso editorial de las revistas científicas. Sin embargo, hace algunos años las investigaciones cualitativas pasaron a tener un papel esencial en la investigación de los fenómenos, pues ayudan a comprender su significado individual y colectivo para la vida de las personas, por lo que se entiende que la tendencia sea a aumentar su representación en las próximas décadas. 
En relación con los participantes de las investigaciones recuperadas, Dall'Acqua y Zaniollo (2009), en un estudio acerca de las tendencias de investigación de los programas de posgrado en educación escolar (PPGEE/UNESP), al igual que se observa en el presente estudio, también refleja que son las muestras de alumnos las que tienen mayor incidencia en las publicaciones. Es posible, entonces, preguntarse si el elevado número de investigaciones con este tipo de personas refleja que las DA están siendo estudiadas sólo en relación con la persona que aprende, aun sabiendo que, de acuerdo con la literatura científica, también ha de tenerse en cuenta la influencia del ambiente físico y social. Se evidencia, así, la necesidad de ampliar el número de investigaciones que atiendan también a los contextos, como la escuela o la familia, con el fin de ampliar el universo de análisis (Robledo y García, 2009; Rueda, 2013).

Con respecto a la distribución de los estudios según el tópico de interés de los mismos, la mayor parte de los trabajos centran su interés en el abordaje de los requisitos necesarios para aprender a leer y escribir. Esto puede justificarse si se tiene en cuenta que la literatura científica sostiene que el principal problema de los estudiantes con DA está relacionado con dificultades en la lectura (Mastropieri, Scruggs y Graetz, 2003); es más, el 90\% de los alumnos con DA muestran dificultades significativas para aprender a leer (Lyon, 1995; Vaughn, Levy, Coleman y Bos, 2002). De esta forma, los alumnos con DA presentan déficits no sólo en las habilidades básicas para la lectura como, por ejemplo, en conciencia fonémica o fonológica, sino también en el análisis del contexto de la palabra y en la interpretación y comprensión de los textos (Sencibaugh, 2007). Seguramente esto hace que se ponga mucho más énfasis en el estudio de la lectura y escritura que en otras áreas, y son muy pocos los trabajos que se interesan en profundizar acerca de los requisitos necesarios para el aprendizaje del área de las Matemáticas. Por otro lado, Klein, Johnson y Ragland (1997), Taylor, Pearson, Clark y Walpole (2000) y Snow, Griffin y Burns (2005) inciden en la importancia de la recolección sistemática de la información en las evaluaciones para analizar el progreso de los alumnos y potenciar la toma de decisiones eficaces en cuanto a las estrategias de enseñanza e intervención. Así, se considera que el número de publicaciones relacionadas con este tópico en la última década es limitado teniendo en cuenta la relevancia del asunto para la identificación de las DA y para poder plantear posibles intervenciones. Los artículos que centran el estudio en la epidemiología, etiología, descripción del cuadro clínico, comorbilidad, diagnóstico y factores de riesgo relacionados con las DA parecen tender a la patologización de la educación. Collares y Moysés (1994) definen esta tendencia como un intento de explicar el fracaso escolar por medio de un reduccionismo biológico, anulando los determinantes políticos y pedagógicos de éste $y$, por tanto, eximiendo al sistema social vigente y a la institución escolar de sus responsabilidades.

Con respecto al resto de tópicos, cabe destacar que un número razonable de investigaciones enfatizan la importancia del autoconcepto y autoestima del alumnado con DA. Sin embargo, aunque es igualmente importante el desarrollo psicomotor, sensorial y perceptivo de los alumnos con DA, parece que ha sido un tópico poco analizado, sobre todo, teniendo en cuenta que algunos autores, como Correia $(1991,2004)$ y la National Joint Committee on Learning Disabilities $(1994,2011)$, matizan que los alumnos con DA presentan trastornos perceptivos y motores, aspectos significativamente relacionados con el proceso de aprendizaje. Por otro lado, los problemas de conducta en DA ha sido también un tópico poco estudiado. Aunque en ocasiones se ha establecido una relación errónea entre dificultades del aprendizaje y problemas de comportamiento del alumno, no se puede considerar que ambos factores sean determinantes entre sí. Por eso, es lógico que el número de artículos encontrados sobre este tópico sea limitado. Por último, es importante reflejar que pocos estudios han centrado la investigación en torno a la intervención en DA, por lo que parece necesario realizar más in- vestigaciones relacionadas con la búsqueda de estrategias que minimicen los efectos de las DA en el aprendizaje.

La distribución de las investigaciones en cuanto al año de publicación, en la última década, mostró un claro crecimiento, hecho que seguramente ha contribuido, positivamente, a la mejora de la comprensión de las dificultades del aprendizaje, entendiendo que cuantos más estudios se realizan sobre esta temática, mejor se puede conocer el fenómeno. Aunque para aclarar los equívocos o las inconsistencias relacionadas con las DA es imprescindible profundizar, aún más, en su estudio. Para empezar, sería importante destacar la necesidad de analizar en otras investigaciones el uso del término dificultades del aprendizaje, ya que no parece haber un claro consenso en la literatura. De hecho, en este estudio se han recuperado más artículos que utilizan otros términos que, propiamente, el término DA. Siendo así, cabe preguntarse si esta falta de coordinación puede, en cierta forma, influir en el uso indiscriminado de los términos y en por qué no se sigue el uso de la nomenclatura adecuada de acuerdo con los documentos de referencia. Por ello, es preciso realizar más estudios que discutan acerca de la terminología dificultad, trastorno o problema, buscando similitudes y diferencias entre las palabras para lograr una unificación en la terminología adoptada. En general, hay una gran variedad de tópicos que se relacionan y superponen con las DA, aunque la mayoría de ellos están relacionados con las de la lectura y escritura. Por ello, debería ampliarse el estudio hacia otros tópicos como, por ejemplo, el aprendizaje de las matemáticas o el desarrollo psicomotor, sensorial y perceptivo. Podrían estudiarse más en profundidad, también, aspectos asociados al contexto escolar en relación con la promoción del aprendizaje: estrategias de aprendizaje, dificultades de los profesores a la hora de enfrentarse a alumnos con DA, recursos y materiales disponibles para desarrollar un trabajo eficaz, entre otros. Por otro lado, se constata en la presente investigación el énfasis en el estudio sobre la persona que aprende, ya que la mayoría de los participantes de las investigaciones son alumnos con DA. Por tanto, sería necesario ampliar el estudio sobre otras cuestiones como, por ejemplo, dónde se aprende o cómo se aprende, promoviendo un entendimiento más amplio de las DA, que tenga en cuenta que la familia, la escuela y las condiciones socioambientales también son factores importantes en la comprensión de esta problemática. En relación con la variedad de diseños metodológicos utilizados, es posible reflejar que la mayoría de los estudios analizados son descriptivos. Sin embargo, no amplían el conocimiento acerca de la prevalencia de los alumnos con DA en las escuelas. Por otro lado, los estudios con diseños cuasi-experimentales recuperados, que representan intervenciones vinculadas a componentes del aprendizaje y situaciones específicas, no parecen facilitar la réplica en el ambiente escolar. Dado que las DA surgen en un ambiente académico, sería importante que la investigación cuasi-experimental se orientara a lograr esa trasferencia con el fin de satisfacer las necesidades particulares de estos alumnos.

Por último, es importante reflejar ciertas limitaciones que han de tenerse en consideración en el presente estudio. Por un lado, no se han tenido en cuenta los posibles sesgos de publicación relacionados con la menor publicación o menor presencia en las bases de datos de estudios con resultados negativos o en contra de las hipótesis de partida y, por otro, tampoco se ha tenido en cuenta como criterio de inclusión o exclusión la calidad de cada uno de los estudios originales utilizados en el presente trabajo. Además, los trabajos recogidos se han limitado a la búsqueda realizada en tres bases de datos concretas, por lo que no se han tenido en cuenta posibles investigaciones que cumplen con los criterios de inclusión, pero que tal vez estén recogidas en bases de datos no consideradas. Tal vez sería interesante en el futuro realizar nuevas revisiones en esta línea que profundicen en estas cuestiones, ampliando la búsqueda a un número más representativo de bases de datos y controlando los criterios de calidad de los estudios seleccionados; por ejemplo, el número de citas recibidas. 


\section{Referencias bibliográficas}

American Psychiatric Association. (2002). Manual Diagnóstico e Estatístico de Transtornos Mentais (DSM-IV-TR). Tradução de M. R. Jorge. 4. . ed., revisada. Porto Alegre: ARTMED.

Bandeira, M., Silva, S., Magalhães, T., Pereira, Z. A., y Del Pettre, A. (2006). Comportamentos problemáticos em estudantes do ensino fundamental: características da ocorrência e relação com habilidades sociais e dificuldades de aprendizagem. Estudos de Psicologia, 11(2), 199-208.

Bastos, O. M., y Deslandes, S. F. (2008). A experiência de ter um filho com deficiência mental: narrativas de mães. Cadernos de Saúde Pública, 24(9), 2141-2150.

Cabral-Filho, J. E. (2006). Os problemas dos Artigos de Revisão. Revista Brasileira de Saúde Materno Infantil, 6(1), 9.

Campos, M. M. (2009). Para que serve a pesquisa em educação? Cadernos de Pesquisa, 39, 269-283.

Capellini, S. A., Conrado T. L. B. C., Tegeiro, M. G., Cera, M. L., Gonçalves, M. P. S., y Ferreira, A. L. B. (2007). Dificuldades de Aprendizagem: manual de orientação para professores e informativo para familiares. Marília: Fundepe Editora.

Carrera, G. (2009). Dificuldades de Aprendizagem. Detecção e estratégias de ajuda prática. Pirapózinho - SP: Grupo Cultural.

Collares, C. A. L., y Moysés, M. A. A. (1994). A transformação do espaço pedagógico em espaço clínico: a patologização da educação. En M. L. Alves (Coord.), Cultura e saúde na escola (pp. 25-31). São Paulo: Fundação para o Desenvolvimento da Educação.

Cook D. J., Mulrow C. D., Haynes R. B. (1997). Systematic reviews: synthesis of best evidence for clinical decisions. Annals of Internal Mdicine, 126, 376-380.

Correia, L. M. (1991). Dificuldades de aprendizagem: contributos para a clarificação e unificação de conceitos. Porto: Associação dos Psicólogos Portugueses.

Correia, L. M. (2004). Problematização das dificuldades de aprendizagem nas necessidades educativas especiais. Análise Psicológica, 22(2), 369-376.

Correia, L. M. (2007). Para uma definição portuguesa de dificuldades de aprendizagem específicas. Revista Brasileira de Educação Especial, 13(7), 155-172.

Dall`Acqua, M. J. C., y Zaniolo, L. O. (2009). Dissertações e teses sobre a temática da educação especial no programa de Pós-Graduação em educação escolar: análise inicial das tendências de pesquisa. Plures. Humanidades, 11, 86-103.

Feldman, R. S. (2005). Psicología: con aplicaciones en países de habla hispana. 6. e․ ed. México: McGrawHill.

Ferreira, W. (2006). Inclusão ou exclusão: reflexões sobre a formação docente dez anos após Salamanca. En D. A. Rodrigues (Org.), Inclusão e Educação. Doze olhares sobre a educação inclusive (pp. 213-238). São Paulo: Summus Editoral.

França, C. (1996). Um novato na Psicopedagogia. En F. Sisto, G. Oliveira, L. Fini, M. Souza y R. Brenelli (Coords.), Atuação psicopedagógica e aprendizagem escolar (pp. 95-109). Petrópolis, RJ: Vozes.

Goodwin, J. C. (1995). Research in Psychology: Methods and design. New York: John Wiley.

Hallahan, D. P., y Kauffman, J. M. (2005). Exceptional learners: introduction to special education. 9.. ed. Boston: Allyn and Bacon.

Higgins, J. P. T., y Green, S. (2011). Cochrane Handbook for Systematic Reviews of Interventions. Version 5.1.0. The Cochrane Collaboration. Recuperado el 19 de noviembre de 2012 de http://www.cochrane-handbook.org

Klein, L., Johnson, J., y Ragland, M. (1997). Successful Texas schoolwide programs: Research study results. Austin, TX: Austin: University of Texas at Austin, The Charles A. Dana Center.

Lyon, G. R. (1995). Research initiatives in LD: Contributions from scientists supported by the National Institute of Child Health and Development. Journal of Child Neurology, 10(suppl. 1), 120-126.
Marturano, E. M., y Loureiro, S. R. (2003). O desenvolvimento socioemocional e as queixas escolares. En A. Del Prette, y Z. A. P. Del Prette (Orgs.), Habilidades sociais, desenvolvimento e aprendizagem: questões conceituais, avaliação e intervenção (pp. 259-291). Campinas: Alínea.

Mastropieri, M. A., Scruggs, T. E., y Graetz, J. E. (2003). Reading comprehension instruction for secondary students: Challenges for struggling students and teachers. Learning Disability Quarterly, 26(4), 103-116.

Mastropieri, M. A., Berkeley, S., Mcduffie, K. A., Graff, H., Marshak, L., Conners, N. A., et al. (2009). What is published in the field of special education? An analysis of 11 prominent journals. Except Child, 76(1), 95-110.

Ministério da Educação (2007). Política Nacional de Educação Especial na Perspectiva da Educação inclusiva. Recuperado el 18 de noviembre de 2012 de http://portal.mec. gov.br/seesp/arquivos/pdf/politica.pdf.

Moojen, S. M. P. (2012). Dificuldades ou transtornos de aprendizagem? En E. Rubinstein (Org.). Psicopedagogia: uma prática, diferentes estilos (pp. 217-254). São Paulo: Casa do Psicólogo.

National Joint Committee on Learning Disabilities. (1994). Collective perspectives on issues affecting learning disabilities. Austin, TX: PRO-ED.

National Joint Committee on Learning Disabilities. (2011). Learning disabilities: implications for policy regarding research and practice: A Report by the National Joint Committee on Learning Disabilities. Learning Disability Ouarterly, 34, 237-241.

Rangel, M. (2009). Educação e saúde: uma relação humana, política e didática. Educação, 32(1), 59-64.

Robledo, P., y García, J. N. (2009). El entorno familiar y su influencia en el rendimiento académico de los alumnos con dificultades de aprendizaje: revisión de estudios empíricos. Aula Abierta, 37(1), 117-128.

Rother, E. T. (2007). Revisión sistemática x revisión narrativa. Acta Paulista de Enfermagem, 20,v-vi.

Rueda, M. I. (2013). La intervención en las dificultades lectoescritoras desde un enfoque multidimensional. Aula Abierta, 41(1), 27-38.

Sencibaugh, J. M. (2007). Meta-analysis of reading comprehension interventions for students with learning disabilities: Strategies and implications. Reading Improvement, 44(1), 6-22.

Shadish, W. R. (2001). Quasi-Experimental Designs. En N. J. Smelser, y P. B. Baltes (Orgs.), International Encyclopedia of the Social y Behavioral Sciences (pp. 12655-12659). Pergamon: Oxford.

Silva, N. L. P, y Dessen, M. A. (2001). Deficiência mental e família: implicações para o desenvolvimento da criança. Psicologia: Teoria e Pesquisa, 17(2), 133-114.

Sisto, F. F. (2001). Dificuldades de aprendizagem. En F. F. Sisto, E. Boruchovitch, L. D. T. Fini, R. P. Brenelli, y S. C. Martinelli (Orgs.), Dificuldades de aprendizagem no contexto psicopedagógico (pp. 19-39). Petrópolis: Vozes.

Snow, C. E., Griffin, P., y Burns, M. S. (2005). Knowledge to support the teaching of reading: preparing teachers for a changing world. San Francisco: Jossey Bay.

Taylor, B. M., Pearson, P. D., Clark, K., y Walpole, S. (2000). Effective schools and accomplished teachers: Lessons about primary grade reading instruction in low-income schools. Elementary School Journal, 101(2), 121-166.

Thomas, J. R., y Nelson, J. K. (1996). Research methods in physical activity. 3. ${ }^{a}$ ed, revisada. Champaign: Human Kinetics.

Turato, E. R. (2005). Métodos qualitativos e quantitativos na área da saúde: definições, diferenças e seus objetos de pesquisa. Rev Saúde Pública, 39(3), 507-514.

Vaughn, S., Levy, S., Coleman, M. y Bos, C. S. (2002). Reading instruction for students with LD and EBD: A synthesis of observation studies. Journal of Special Education, 36(1), 2-13.

Werner, E. E. (1998). Protective factors and individual resilience. En S. J. Meisels y J. P. Shonkoff (Orgs), Handbook of early childhood intervention (pp. 97-116). Nueva York: Cambridge University. 